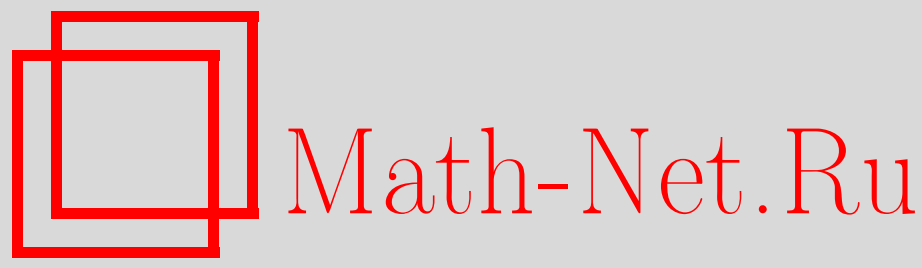

Р. Р. Мецаев, БРСТ-БВ-подход к безмассовым полям, адаптированный для АдС/КТП-соответствия, ТMФ, 2016, том 187, номер 2, 323-337

DOI: https://doi.org/10.4213/tmf9030

Использование Общероссийского математического портала Math-Net.Ru подразумевает, что вы прочитали и согласны с пользовательским соглашением http: //www . mathnet.ru/rus/agreement

Параметры загрузки:

IP : 54.89 .56 .158

26 апреля 2023 г., 05:48:17

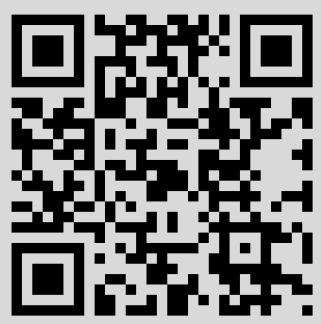




\title{
БРСТ-БВ-ПОДХОД К БЕЗМАССОВЫМ ПОЛЯМ, АДАПТИРОВАННЫЙ ДЛЯ АДС/КТП-СООТВЕТСТВИЯ
}

\begin{abstract}
В рамках БРСТ-БВ-подхода к формулировке релятивистской динамики рассмотрены безмассовые и массивные поля произвольного спина, распространяющиеся в плоском пространстве, а также безмассовые поля произвольного спина, распространяющиеся в пространстве АдС. Для таких полей получены БРСТ-БВ-лагранжианы, инвариантные относительно калибровочных преобразований. Лагранжианы и калибровочные преобразования построены в терминах бесследовых калибровочных полей и бесследовых параметров калибровочных преобразований. Поля в пространстве АдС рассмотрены с применением пуанкаре-параметризации этого пространства, что приводит к простому виду БРСТ-БВ-лагранжиана. Показано, что в калибровке Зигеля лагранжиан безмассовых полей АдС приводит к расщепляющимся уравнениям движения и это существенно упрощает исследование АдС/КТП-соответствия. В базисе конформной алгебры найдена реализация релятивистских симметрий на полях и антиполях в пространстве АдС.
\end{abstract}

Ключевые слова: БРСТ-симметрия, поля высших спинов, АдС/КТП-соответствие.

DOI: $10.4213 / \operatorname{tmf} 9030$

\section{1. ВВЕДЕНИЕ}

БРСТ-подход [1] возник как метод, который упростил анализ тождеств СлавноваТейлора [2] в калибровочных теориях. В настоящее время БРСТ-подход является основным методом исследования ультрафиолетовых расходимостей и построения релятивистски-инвариантной перенормированной $S$-матрицы в калибровочных теориях. Более того, расширенная версия БРСТ-подхода, которая включает антиполя, стала мощным средством исследования не только теорий квантованных калибровочных полей, но и эффективным методом построения теорий классических калибровочных полей [3]. В настоящей работе именно расширенную версию БРСТ-подхода мы называем БРСТ-БВ-подходом к формулировке релятивистской динамики.

Работа выполнена при финансовой поддержке РФФИ (грант № 14-02-01171).

*Физический институт им. П. Н. Лебедева РАН, Москва, Россия. E-mail: metsaev@lpi.ru 
Теория безмассовых полей высших спинов [4] в пространстве анти-де Ситтера (АдС) является, с одной стороны, интересным кандидатом на роль теории фундаментальных взаимодействий. С другой стороны, гипотеза дуальности Малдасены не только привела к новому всплеску активного интереса к теории струн, но и открыла новые интересные приложения теорий безмассовых полей высших спинов для изучения конформных теорий. Основным объектом, который устанавливает связь между теориями полей в пространстве АдС и конформными теориями, является так называемое эффективное действие, определяемое как континуальный интеграл по полям в пространстве АдС с заданными граничными значениями для полей на границе пространства АдС. Граничные значения полей определяются как источники, а вариационные производные эффективного действия по источникам задают корреляционные функции конформной теории, которая дуальна теории поля в пространстве АдС. Таким образом, проблема вычисления эффективного действия является актуальной для изучения гипотезы дуальности Малдасены, которую часто называют АдС/КТП-соответствием.

Проблеме вычисления эффективного действия уделялось много внимания в силу ее важности при исследовании АдС/КТП-соответствия. В квадратичном приближении по источникам древесное эффективное действие для безмассовых полей спина $s=1, s=2$ и произвольного спина было вычислено в работах [5]-[7] соответственно, а древесное эффективное действие для массивных полей спина $s=1$, $s=2$ и произвольного спина было исследовано в статьях [8]-[10] соответственно (см. также работу [11]). Исследование теоретико-групповых аспектов, связанных с проблемой вычисления эффективного действия, можно найти в статьях [12]-[14]. Вычисление $n$-точечных древесных корреляторов безмассовых полей высших спинов обсуждается в работах [15] (см. также недавние работы [16]). Различные аспекты однопетлевого квантового эффективного действия изучаются в статьях [17].

В работе [18] мы начали исследование эффективного действия с помощью БРСТподхода. А именно, используя метрический формализм полей высших спинов в пространстве АдС, а также модифицированную калибровку де Дондера, найденные в статьях [19], [20], и применив в работе [18] стандартную процедуру Фаддеева-Попова, мы получили простое действие полей высших спинов, инвариантное относительно глобальных БРСТ-преобразований, и использовали данное действие для исследования АдС/КТП-соответствия. В настоящей статье мы получаем простое БРСТ-БВ-действие безмассовых полей высших спинов, которое инвариантно относительно локальных калибровочных симметрий и адаптировано для изучения АдС/КТП-соответствия. Действие, рассмотренное в работе [18], получено из БРСТБВ-действия, найденного в данной работе с применением калибровки Зигеля.

Разрабатываемые далее БРСТ-БВ-формулировки полей в пространствах $\mathbb{R}^{d-1,1}$ и $\mathrm{AдC}_{d+1}$ имеют некоторые общие черты. Поэтому в разделе 2 мы начинаем обсуждение БРСТ-БВ-формулировок для безмассовых и массивных полей произвольного спина на примере более простого случая пространства Минковского. В разделе 3, используя пуанкаре-параметризацию пространства АдС, мы получаем БРСТ-БВдействие безмассовых полей произвольного спина в пространстве АдС, адаптированное для изучения АдС/КТП-соответствия. Также мы получаем реализацию релятивистских симметрий алгебры $s o(d, 2)$ на калибровочных полях и антиполях в рамках БРСТ-БВ-подхода. 


\section{2. БЕЗМАССОВЫЕ И МАССИВНЫЕ ПОЛЯ В ПРОСТРАНСТВЕ $\mathbb{R}^{d-1,1}$}

В настоящем разделе мы рассматриваем БРСТ-БВ-формулировки на примерах безмассовых и массивных полей, распространяющихся в пространстве Минковского $\mathbb{R}^{d-1,1}$

Безмассовые поля. Состав полей, необходимый для построения БРСТ-БВформулировки, удобно ввести, используя генерирующую форму описания полей. Чтобы определить генерирующую форму полей, введем грассманову переменную $\theta$, грассманово-четные осцилляторы $\alpha^{a}$ и нечетные осцилляторы $\eta, \rho$. Осцилляторы $\alpha^{a}$ преобразуются как векторы алгебры Лоренца $s o(d-1,1)$, а переменная $\theta$ и осцилляторы $\eta, \rho$ являются скалярами этой алгебры. С помощью $\theta$ и осцилляторов введем кет-вектор

$$
|\Phi\rangle=\Phi(x, \theta, \alpha, \eta, \rho)|0\rangle,
$$

где $x$ - обозначение для координат $x^{a}$ пространства $\mathbb{R}^{d-1,1}$. Обычные поля, зависящие от $x^{a}$, получаются разложением грассманово-четного поля $\Phi(2.1)$ в ряд Тейлора по грассмановой координате $\theta$ и осцилляторам $\alpha^{a}, \eta, \rho$. Для описания безмассового поля спина $s$ мы налагаем следующие алгебраические ограничения на кет-вектор $|\Phi\rangle(2.1)$ :

$$
\begin{aligned}
\left(N_{\alpha}+N_{\eta}+N_{\rho}-s\right)|\Phi\rangle & =0, \\
\bar{\alpha}^{2}|\Phi\rangle & =0 .
\end{aligned}
$$

Определение операторов $N_{\alpha}, N_{\eta}, N_{\rho}$ и $\bar{\alpha}^{2}$ можно найти в приложении настоящей статьи (см. формулы (П.1), (П.2)). Ограничение (2.2) означает, что кет-вектор $|\Phi\rangle$ является однородным полиномом степени $s$ относительно осцилляторов $\alpha^{a}, \eta, \rho$, а ограничение (2.3) означает, что поля, которые получаются при разложении генерирующего поля $\Phi(2.1)$ в ряд Тейлора по осцилляторам $\alpha^{a}$, являются бесследовыми тензорными полями алгебры Лоренца $s o(d-1,1)$.

Чтобы прояснить состав тензорных полей, включенных в кет-вектор $|\Phi\rangle$ (2.1), заметим, что разложение кет-вектора $|\Phi\rangle$ в ряд Тейлора по грассмановой переменной $\theta$ и грассманово-нечетным осцилляторам $\eta, \rho$ можно представить следующим образом:

$$
\begin{aligned}
|\Phi\rangle & =|\phi\rangle+\theta\left|\phi_{*}\right\rangle, \\
|\phi\rangle & =\left|\phi_{\mathrm{I}}\right\rangle+\rho|c\rangle+\eta|\bar{c}\rangle+\rho \eta\left|\phi_{\mathrm{II}}\right\rangle, \\
\left|\phi_{*}\right\rangle & =\left|\phi_{\mathrm{I} *}\right\rangle+\rho\left|\bar{c}_{*}\right\rangle+\eta\left|c_{*}\right\rangle+\rho \eta\left|\phi_{\mathrm{II} *}\right\rangle .
\end{aligned}
$$

Кет-векторы, появляющиеся в правых частях соотношений (2.5), (2.6), зависят от векторных осцилляторов $\alpha^{a}$. Рассмотрим подробнее кет-векторы в формуле (2.5). Учитывая равенство (2.2), находим, что кет-векторы (2.5) зависят от осцилляторов $\alpha^{a}$ следующим образом:

$$
\begin{aligned}
\left|\phi_{\mathrm{I}}\right\rangle & =\frac{1}{s !} \alpha^{a_{1}} \ldots \alpha^{a_{s}} \phi_{\mathrm{I}}^{a_{1} \ldots a_{s}}(x)|0\rangle, \\
|c\rangle & =\frac{1}{(s-1) !} \alpha^{a_{1}} \ldots \alpha^{a_{s-1}} c^{a_{1} \ldots a_{s-1}}(x)|0\rangle,
\end{aligned}
$$




$$
\begin{aligned}
|\bar{c}\rangle & =\frac{1}{(s-1) !} \alpha^{a_{1}} \ldots \alpha^{a_{s-1}} \bar{c}^{a_{1} \ldots a_{s-1}}(x)|0\rangle, \\
\left|\phi_{\mathrm{II}}\right\rangle & =\frac{1}{(s-2) !} \alpha^{a_{1}} \ldots \alpha^{a_{s-2}} \phi_{\mathrm{II}}^{a_{1} \ldots a_{s-2}}(x)|0\rangle .
\end{aligned}
$$

Из соотношений (2.7)-(2.10) получим тензорные поля, которые определяют кет-векторы (2.5). А именно, мы видим, что есть одно тензорное поле $\phi_{\mathrm{I}}^{a_{1} \ldots a_{s}}$ ранга $s$, два тензорных поля $c^{a_{1} \ldots a_{s-1}}, \bar{c}^{a_{1} \ldots a_{s-1}}$ ранга $s-1$ и одно тензорное поле $\phi_{\mathrm{II}}^{a_{1} \ldots a_{s-2}}$ ранга $s-2$. Эти тензорные поля симметричны относительно перестановок своих тензорных индексов. Ограничение (2.3) означает, что данные тензорные поля являются бесследовыми. Заметим, что кет-вектор $\left|\phi_{*}\right\rangle(2.6)$ можно представить с помощью тензорных антиполей в виде, аналогичном соотношениям (2.7)-(2.10). Итак, рассматриваемые кет-векторы представимы в терминах бесследовых симметричных тензорных полей алгебры Лоренца $s o(d-1,1)$.

БРСТ-БВ-лагранжиан. При использовании БРСТ-БВ-подхода общее представление для калибровочно-инвариантного действия для полей в пространстве $\mathbb{R}^{d-1,1}$ имеет вид [3]

$$
S=\int d^{d} x \mathcal{L}, \quad \mathcal{L}=\frac{1}{2} \int d \theta\langle\Phi|Q| \Phi\rangle .
$$

БРСТ-оператор $Q$, задающий лагранжиан (2.11), допускает следующее представление:

$$
Q=\theta\left(\square-M^{2}\right)+M^{\eta a} \partial^{a}+M^{\eta}+M^{\eta \eta} \partial_{\theta}
$$

где $\square=\partial^{a} \partial^{a}$ - оператор Даламбера в пространстве $\mathbb{R}^{d-1,1}$, а $\partial_{\theta}$ означает левую производную по грассмановой переменной: $\partial_{\theta}=\partial / \partial \theta$. Как видно из формулы $(2.12)$, БРСТ-оператор $Q$ определяется операторами $M^{2}, M^{\eta a}, M^{\eta}$ и $M^{\eta \eta}$. Эти операторы зависят от осцилляторов и не зависят от грассмановой координаты $\theta$, координат $x^{a}$ пространства $\mathbb{R}^{d-1,1}$ и производных $\partial_{\theta}, \partial^{a}$. Оператор $M^{2}$ является квадратом оператора массы. Операторы $M^{\eta a}, M^{\eta}$ и $M^{\eta \eta}$ назовем спиновыми операторами. Условие $Q^{2}=0$ приводит к (анти)коммутаторам для спиновых операторов и оператора $M^{2}$ :

$$
\begin{aligned}
& \left\{M^{\eta a}, M^{\eta b}\right\}=-2 \eta^{a b} M^{\eta \eta}, \quad\left\{M^{\eta}, M^{\eta}\right\}=2 M^{2} M^{\eta \eta}, \\
& {\left[M^{2}, M^{\eta a}\right]=0, \quad\left[M^{2}, M^{\eta}\right]=0, \quad\left[M^{2}, M^{\eta \eta}\right]=0,} \\
& \left\{M^{\eta a}, M^{\eta}\right\}=0, \quad\left[M^{\eta a}, M^{\eta \eta}\right]=0, \quad\left[M^{\eta}, M^{\eta \eta}\right]=0 .
\end{aligned}
$$

Калибровочные преобразования, относительно которых действие (2.11) инвариантно, задаются соотношением

$$
\delta|\Phi\rangle=Q|\Xi\rangle
$$

где кет-вектор $|\Xi\rangle$ определяет генерирующую форму параметров этих преобразований. Данный кет-вектор зависит от грассмановой координаты $\theta$, осцилляторов $\alpha^{a}$, $\eta, \rho$ и удовлетворяет тем же алгебраическим соотношениям, что и кет-вектор $|\Phi\rangle$ :

$$
\begin{aligned}
|\Xi\rangle & =\Xi(x, \theta, \alpha, \eta, \rho)|0\rangle, \\
\left(N_{\alpha}+N_{\eta}+N_{\rho}-s\right)|\Xi\rangle & =0, \\
\bar{\alpha}^{2}|\Xi\rangle & =0,
\end{aligned}
$$


где $\Xi$ в формуле (2.15) является грассманово-нечетным. Так как $|\Phi\rangle$ и $|\Xi\rangle$ удовлетворяют одним и тем же алгебраическим ограничениям, представление $|\Xi\rangle$ в терминах параметров калибровочных преобразований, которые суть симметричные бесследовые тензоры алгебры Лоренца $s o(d-1,1)$, имеет тот же вид, что и представление $|\Phi\rangle$ в терминах тензорных калибровочных полей (см. соотношения (2.4)-(2.10)).

Итак, построение калибровочно-инвариантного действия и соответствующих калибровочных преобразований сводится к проблеме построения БРСТ-оператора. В свою очередь, построение БРСТ-оператора сводится к проблеме поиска реализации спиновых операторов, удовлетворяющих соотношениям (2.13). В рамках рассматриваемого подхода безмассовое поле описывается кет-вектором (2.1), который удовлетворяет ограничениям (2.2) и (2.3). Это означает, что мы должны построить реализацию спиновых операторов на пространстве кет-вектора (2.1). Получаем следующую реализацию для спиновых операторов и оператора $M^{2}$ :

$$
\begin{aligned}
M^{\eta a} & =\eta g_{\rho} \bar{\alpha}^{a}+A^{a} \bar{g}_{\eta} \bar{\eta}, \\
M^{\eta \eta} & =\eta \bar{\eta}, \\
M^{\eta} & =0, \\
M^{2} & =0,
\end{aligned}
$$

где использованы обозначения

$$
A^{a} \equiv \alpha^{a}-\alpha^{2} \frac{1}{2 N_{\alpha}+d} \bar{\alpha}^{a}, g_{\rho} \equiv\left[\frac{2 s+d-4-2 N_{\rho}}{2 s+d-4}\right]^{1 / 2}, \bar{g}_{\eta} \equiv-\left[\frac{2 s+d-4-2 N_{\eta}}{2 s+d-4}\right]^{1 / 2} .
$$

Справедливо следующее замечание.

ЗАмечАниЕ 2.1. Ограничение (2.2) хорошо известно в литературе. Ранее в ней вместо (2.3) использовалось ограничение (см., например, работы [21])

$$
\left(\bar{\alpha}^{2}+2 \bar{\eta} \bar{\rho}\right)|\Phi\rangle=0 .
$$

При использовании формулы (2.22) соотношения (2.19)-(2.21) не меняются, в то время как оператор $M^{\eta a}$ принимает вид $M^{\eta a}=\eta \bar{\alpha}^{a}-\alpha^{a} \bar{\eta}$. В данной статье мы предлагаем использовать ограничение (2.3), так как оно представляется нам удобным для построения БРСТ-БВ-формулировки полей в пространстве АдС, адаптированной для исследования АдС/КТП-соответствия. Изучение БРСТ-формулировок без использования условий бесследовости можно найти в работах [22]. Игнорирование условий бесследовости для кет-вектора (2.1) приводит к БРСТ-формулировке так называемых приводимых триплетных мультиплетов высших спинов [23].

Массивные поля. Для построения БРСТ-БВ-действия мы используем генерирующую форму описания полей, которую мы введем с помощью грассмановой переменной $\theta$, грассманово-четных осцилляторов $\alpha^{a}, \zeta$ и нечетных осцилляторов $\eta, \rho$. Осцилляторы $\alpha^{a}$ преобразуются как векторы алгебры Лоренца $s o(d-1,1)$, а $\theta$ и осцилляторы $\zeta, \eta, \rho$ являются скалярами алгебры Лоренца. С помощью грассмановой переменной и осцилляторов определим кет-вектор

$$
|\Phi\rangle=\Phi(x, \theta, \alpha, \zeta, \eta, \rho)|0\rangle
$$


который удовлетворяет следующим алгебраическим ограничениям:

$$
\begin{aligned}
\left(N_{\alpha}+N_{\zeta}+N_{\eta}+N_{\rho}-s\right)|\Phi\rangle & =0, \\
\bar{\alpha}^{2}|\Phi\rangle & =0 .
\end{aligned}
$$

По определению поле $\Phi$ (2.23) является грассманово-четным. Отметим, что ограничение (2.25) имеет тот же вид, что и ограничение (2.3) для безмассовых полей.

Чтобы прояснить состав тензорных полей, включенных в кет-вектор $|\Phi\rangle$ (2.23), заметим, что разложение $|\Phi\rangle$ в ряд по $\theta, \eta, \rho$ можно записать так же, как это сделано в соотношениях (2.4)-(2.6). Заметим однако, что для массивных полей кет-векторы, появляющиеся в правых частях соотношений $(2.5),(2.6)$, зависят не только от векторных осцилляторов $\alpha^{a}$, но также и от осциллятора $\zeta$. Для примера рассмотрим кет-векторы в формуле (2.5). Учитывая соотношение (2.24), находим, что кет-векторы (2.5) зависят от осцилляторов $\alpha^{a}, \zeta$ следующим образом:

$$
\begin{aligned}
\left|\phi_{\mathrm{I}}\right\rangle & =\sum_{s^{\prime}=0}^{s} \frac{\zeta^{s-s^{\prime}}}{\sqrt{\left(s-s^{\prime}\right) !}\left|\phi_{\mathrm{I}}^{s^{\prime}}\right\rangle,} & \left|\phi_{\mathrm{I}}^{s^{\prime}}\right\rangle & =\frac{1}{s^{\prime} !} \alpha^{a_{1}} \ldots \alpha^{a_{s^{\prime}}} \phi_{\mathrm{I}}^{a_{1} \ldots a_{s^{\prime}}}(x)|0\rangle, \\
|c\rangle & =\sum_{s^{\prime}=0}^{s-1} \frac{\zeta^{s-1-s^{\prime}}}{\sqrt{\left(s-1-s^{\prime}\right) !}}\left|c^{s^{\prime}}\right\rangle, & \left|c^{s^{\prime}}\right\rangle & =\frac{1}{s^{\prime} !} \alpha^{a_{1}} \ldots \alpha^{a_{s^{\prime}}} c^{a_{1} \ldots a_{s^{\prime}}}(x)|0\rangle, \\
|\bar{c}\rangle & =\sum_{s^{\prime}=0}^{s-1} \frac{\zeta^{s-1-s^{\prime}}}{\sqrt{\left(s-1-s^{\prime}\right) !}}\left|\bar{c}^{s^{\prime}}\right\rangle, & \left|\bar{c}^{s^{\prime}}\right\rangle & =\frac{1}{s^{\prime} !} \alpha^{a_{1}} \ldots \alpha^{a_{s^{\prime}}} \bar{c}^{a_{1} \ldots a_{s^{\prime}}}(x)|0\rangle, \\
\left|\phi_{\mathrm{II}}\right\rangle & =\sum_{s^{\prime}=0}^{s-2} \frac{\zeta^{s-2-s^{\prime}}}{\sqrt{\left(s-2-s^{\prime}\right) !}}\left|\phi_{\mathrm{II}}^{s^{\prime}}\right\rangle, & \left|\phi_{\mathrm{II}}^{s^{\prime}}\right\rangle & =\frac{1}{s^{\prime} !} \alpha^{a_{1}} \ldots \alpha^{a_{s^{\prime}}} \phi_{\mathrm{II}}^{a_{1} \ldots a_{s^{\prime}}}(x)|0\rangle .
\end{aligned}
$$

Кет-вектор $\left|\phi_{*}\right\rangle(2.6)$, отвечающий массивным полям, представим в терминах тензорных антиполей в виде, аналогичном соотношениям (2.26).

Для построения БРСТ-оператора следует найти реализацию спиновых операторов, удовлетворяющих соотношениям (2.13). В рамках предлагаемого подхода массивное поле описывается кет-вектором (2.23), который удовлетворяет соотношениям (2.24), (2.25). Таким образом, мы должны построить реализацию спиновых операторов на пространстве кет-вектора (2.23). Получим следующую реализацию для спиновых операторов и оператора $M^{2}$ :

$$
\begin{aligned}
M^{\eta a} & =\eta g_{\rho \zeta} \bar{\alpha}^{a}+A^{a} \bar{g}_{\eta \zeta} \bar{\eta}, \\
M^{\eta \eta} & =\eta \bar{\eta}, \\
M^{\eta} & =\eta l_{\rho \zeta} \bar{\zeta}+\zeta \bar{l}_{\eta \zeta} \bar{\eta}, \\
M^{2} & =m^{2},
\end{aligned}
$$

где $m$ в $(2.30)$ - параметр массы, и мы используем обозначения

$$
\begin{aligned}
A^{a} & \equiv \alpha^{a}-\alpha^{2} \frac{1}{2 N_{\alpha}+d} \bar{\alpha}^{a}, & g_{\rho \zeta} & \equiv\left[\frac{2 s+d-4-2 N_{\zeta}-2 N_{\rho}}{2 s+d-4-2 N_{\zeta}}\right]^{1 / 2}, \\
\bar{g}_{\eta \zeta} & \equiv-\left[\frac{2 s+d-4-2 N_{\zeta}-2 N_{\eta}}{2 s+d-4-2 N_{\zeta}}\right]^{1 / 2}, & l_{\rho \zeta} & \equiv m e_{\zeta}\left[\frac{2 s+d-4-2 N_{\zeta}-2 N_{\rho}}{2 s+d-4-2 N_{\zeta}}\right] \\
\bar{l}_{\eta \zeta} & \equiv m e_{\zeta}\left[\frac{2 s+d-4-2 N_{\zeta}-2 N_{\eta}}{2 s+d-4-2 N_{\zeta}}\right]^{-1 / 2}, & e_{\zeta} & \equiv\left[\frac{2 s+d-4-N_{\zeta}}{2 s+d-4-2 N_{\zeta}}\right]^{1 / 2} .
\end{aligned}
$$


Калибровочные преобразования массивных полей, относительно которых инвариантно действие (2.11), задаются тем же соотношением, что и преобразование (2.14), где кет-вектор $|\Xi\rangle$ определяет генерирующую форму параметров калибровочных преобразований. Для массивных полей этот кет-вектор зависит от грассмановой координаты $\theta$, осцилляторов $\alpha^{a}, \zeta, \eta, \rho$ и удовлетворяет тем же алгебраическим соотношениям, что и кет-вектор $|\Phi\rangle(2.24),(2.25)$ :

$$
|\Xi\rangle=\Xi(x, \theta, \alpha, \zeta, \eta, \rho)|0\rangle, \quad\left(N_{\alpha}+N_{\zeta}+N_{\eta}+N_{\rho}-s\right)|\Xi\rangle=0, \quad \bar{\alpha}^{2}|\Xi\rangle=0 .
$$

\section{3. БЕЗМАССОВЫЕ ПОЛЯ В ПРОСТРАНСТВЕ АДС ${ }_{d+1}$}

В настоящем разделе мы строим БРСТ-БВ-формулировку безмассового поля спина $s$ в пространстве $\mathrm{AдC}_{d+1}$. Мы используем пуанкаре-параметризацию пространства $\mathrm{AдC}_{d+1}$ :

$$
d s^{2}=\frac{1}{z^{2}}\left(d x^{a} d x^{a}+d z d z\right), \quad a=0,1, \ldots, d-1 .
$$

Из соотношения (3.1) видно, что при использовании пункаре-параметризации только симметрии алгебры $s o(d-1,1)$ реализованы в явном виде.

Состав полей. Состав полей удобно ввести, используя генерирующую форму описания полей, которую мы определим с помощью грассмановой переменной $\theta$, грассманово-четных осцилляторов $\alpha^{a}, \alpha^{z}$ и нечетных осцилляторов $\eta, \rho$. Осцилляторы $\alpha^{a}$ преобразуются как векторы алгебры Лоренца $s o(d-1,1)$, а грассманова переменная $\theta$ и осцилляторы $\alpha^{z}, \eta, \rho$ являются скалярами алгебры Лоренца. С помощью грассмановой переменной и осцилляторов введем кет-вектор

$$
|\Phi\rangle=\Phi\left(x, z, \theta, \alpha, \alpha^{z}, \eta, \rho\right)|0\rangle
$$

где переменные $x, z$ - суть координаты Пуанкаре $x^{a}, z$ пространства $\mathrm{AдC}_{d+1}(3.1)$. По определению поле $\Phi$ (3.2) является грассманово-четным. Тензорные поля, зависящие от координат $x^{a}, z$, можно получить с помощью разложения $|\Phi\rangle(3.2)$ в ряд Тейлора по $\theta$ и осцилляторам $\alpha^{a}, \alpha^{z}, \eta, \rho$. Для описания безмассового поля спина $s$ мы налагаем следующие алгебраические ограничения на кет-вектор $|\Phi\rangle$ (3.2):

$$
\begin{aligned}
\left(N_{\alpha}+N_{z}+N_{\eta}+N_{\rho}-s\right)|\Phi\rangle & =0 \\
\bar{\alpha}^{2}|\Phi\rangle & =0 .
\end{aligned}
$$

Определение операторов $N_{\alpha}, N_{z}, N_{\eta}, N_{\rho}$ и $\bar{\alpha}^{2}$ можно найти в приложении настоящей статьи (см. формулы (П.1), (П.2)). Соотношение (3.3) означает, что кет-вектор $|\Phi\rangle$ является однородным полиномом степени $s$ относительно осцилляторов $\alpha^{a}, \alpha^{z}, \eta, \rho$, а соотношение (3.4) - что поля, которые получаются при разложении $|\Phi\rangle(3.2)$ в ряд Тейлора по осцилляторам $\alpha^{a}$, являются бесследовыми тензорными полями алгебры Лоренца $s o(d-1,1)$.

Чтобы прояснить состав тензорных полей, включенных в кет-вектор $|\Phi\rangle$ (3.2), заметим, что, во-первых, разложение кет-вектора $|\Phi\rangle(3.2)$ в ряд по $\theta, \eta, \rho$ имеет вид, аналогичный соотношениям (2.4)-(2.6). Во вторых, ограничение (3.3) для безмассового поля в пространстве $\mathrm{AдC}_{d+1}$ получается из ограничения (2.24) для массивного поля в пространстве $\mathbb{R}^{d-1,1}$ простой заменой осциллятора $\zeta$ на осциллятор $\alpha^{z}$. Это 
означает, что кет-векторы (2.5) для безмассового поля можно найти с помощью простой замены $\zeta$ на $\alpha^{z}$ в формулах (2.26):

$$
\begin{aligned}
\left|\phi_{\mathrm{I}}\right\rangle & =\sum_{s^{\prime}=0}^{s} \frac{\alpha_{z}^{s-s^{\prime}}}{\sqrt{\left(s-s^{\prime}\right) !}\left|\phi_{\mathrm{I}}^{s^{\prime}}\right\rangle,} & \left|\phi_{\mathrm{I}}^{s^{\prime}}\right\rangle & =\frac{1}{s^{\prime} !} \alpha^{a_{1}} \ldots \alpha^{a_{s^{\prime}}} \phi_{\mathrm{I}}^{a_{1} \ldots a_{s^{\prime}}}(x, z)|0\rangle, \\
|c\rangle & =\sum_{s^{\prime}=0}^{s-1} \frac{\alpha_{z}^{s-1-s^{\prime}}}{\sqrt{\left(s-1-s^{\prime}\right) !}}\left|c^{s^{\prime}}\right\rangle, & \left|c^{s^{\prime}}\right\rangle & =\frac{1}{s^{\prime} !} \alpha^{a_{1}} \ldots \alpha^{a_{s^{\prime}}} c^{a_{1} \ldots a_{s^{\prime}}}(x, z)|0\rangle, \\
|\bar{c}\rangle & =\sum_{s^{\prime}=0}^{s-1} \frac{\alpha_{z}^{s-1-s^{\prime}}}{\sqrt{\left(s-1-s^{\prime}\right) !}}\left|\bar{c}^{s^{\prime}}\right\rangle, & \left|\bar{c}^{s^{\prime}}\right\rangle & =\frac{1}{s^{\prime} !} \alpha^{a_{1}} \ldots \alpha^{a_{s^{\prime}}} \bar{c}^{a_{1} \ldots a_{s^{\prime}}}(x, z)|0\rangle, \\
\left|\phi_{\mathrm{II}}\right\rangle & =\sum_{s^{\prime}=0}^{s-2} \frac{\alpha_{z}^{s-2-s^{\prime}}}{\sqrt{\left(s-2-s^{\prime}\right) !}}\left|\phi_{\mathrm{II}}^{s^{\prime}}\right\rangle, & \left|\phi_{\mathrm{II}}^{s^{\prime}}\right\rangle & =\frac{1}{s^{\prime} !} \alpha^{a_{1}} \ldots \alpha^{a_{s^{\prime}}} \phi_{\mathrm{II}}^{a_{1} \ldots a_{s^{\prime}}}(x, z)|0\rangle .
\end{aligned}
$$

Кет-вектор $\left|\phi_{*}\right\rangle$ (2.6), отвечающий безмассовому полю в пространстве $\mathrm{AдC}_{d+1}$, представим в терминах тензорных антиполей в виде, аналогичном соотношениям (3.5).

БРСТ-БВ-лагранжиан. При применении БРСТ-БВ-подхода общее представление для калибровочно-инвариантного действия имеет вид

$$
S=\int d^{d} x d z \mathcal{L}, \quad \mathcal{L}=\frac{1}{2} \int d \theta\langle\Phi|Q| \Phi\rangle
$$

БРСТ-оператор $Q$, задающий лагранжиан (3.6), допускает следующее представление:

$$
Q=\theta\left(\square-M^{2}\right)+M^{\eta a} \partial^{a}+M^{\eta}+M^{\eta \eta} \partial_{\theta}
$$

где $\square=\partial^{a} \partial^{a}$ - оператор Даламбера в пространстве $\mathbb{R}^{d-1,1}$, а $\partial_{\theta}$ означает левую производную по грассмановой переменной: $\partial_{\theta}=\partial / \partial \theta$. Как видно из равенства (3.7), БРСТ-оператор определяется операторами $M^{2}, M^{\eta a}, M^{\eta}$ и $M^{\eta \eta}$. Все эти операторы зависят от осцилляторов, в то время как операторы $M^{\eta a}, M^{\eta \eta}$ не зависят от грассмановой переменной $\theta$, координат $x^{a}, z$ и производных $\partial_{\theta}, \partial^{a}, \partial_{z}$. Операторы $M^{2}, M^{\eta}$ также не зависят от грассмановой переменной $\theta$, координат $x^{a}$ и производных $\partial_{\theta}, \partial^{a}$, но зависят от радиальной координаты $z$ и производной $\partial_{z}$. Условие $Q^{2}=0$ приводит к (анти)коммутаторам (2.13).

Сравнивая формулы (2.11), (2.12) с равенствами (3.6), (3.7), отметим, что БРСТБВ-действие, которое в настоящей работе мы предлагаем использовать для описания полей в пространстве $\mathrm{AдC}_{d+1}$, по своей общей структуре совпадает с действием для полей в пространстве $\mathbb{R}^{d-1,1}$, предложенным в работах [3]. Заметим также, что именно использование пуанкаре-параметризации в пространстве АдС (3.1) позволяет получить БРСТ-БВ-действие в виде формул (3.6), (3.7).

Для построения БРСТ-оператора $Q$ (3.7) следует найти спиновые операторы, удовлетворяющие соотношениям (2.13). В предлагаемом подходе безмассовое поле описывается кет-вектором (3.2), который удовлетворяет соотношениям (3.3), (3.4). Итак, мы должны построить реализацию спиновых операторов на пространстве 
кет-вектора (3.2). Находим следующую реализацию для спиновых операторов и оператора $M^{2}$ :

$$
\begin{aligned}
M^{\eta a} & =\eta g_{\rho z} \bar{\alpha}^{a}+A^{a} \bar{g}_{\eta z} \bar{\eta} \\
M^{\eta \eta} & =\eta \bar{\eta} \\
M^{\eta} & =\eta l_{\rho z} \bar{\alpha}^{z}+\alpha^{z} \bar{l}_{\eta z} \bar{\eta} \\
M^{2} & =-\partial_{z}^{2}+\frac{1}{z^{2}}\left(\nu^{2}-\frac{1}{4}\right),
\end{aligned}
$$

где операторы $A^{a}, g_{\rho z}, \bar{g}_{\eta z}, l_{\rho z}, \bar{l}_{\eta z}, \nu$ определяются соотношениями

$$
\begin{gathered}
A^{a} \equiv \alpha^{a}-\alpha^{2} \frac{1}{2 N_{\alpha}+d} \bar{\alpha}^{a} \\
g_{\rho z} \equiv\left[\frac{2 s+d-4-2 N_{z}-2 N_{\rho}}{2 s+d-4-2 N_{z}}\right]^{1 / 2}, \quad \bar{g}_{\eta z} \equiv-\left[\frac{2 s+d-4-2 N_{z}-2 N_{\eta}}{2 s+d-4-2 N_{z}}\right]^{1 / 2} \\
l_{\rho z} \equiv \mathcal{T}_{-\nu+1 / 2} e_{z}\left[\frac{2 s+d-4-2 N_{z}-2 N_{\rho}}{2 s+d-4-2 N_{z}}\right]^{-1 / 2} \\
\bar{l}_{\eta z} \equiv-\mathcal{T}_{\nu-1 / 2} e_{z}\left[\frac{2 s+d-4-2 N_{z}-2 N_{\eta}}{2 s+d-4-2 N_{z}}\right]^{-1 / 2} \\
\nu \equiv s+\frac{d-4}{2}-N_{z} \\
e_{z} \equiv\left[\frac{2 s+d-4-N_{z}}{2 s+d-4-2 N_{z}}\right]^{1 / 2} \\
\mathcal{T}_{\nu} \equiv \partial_{z}+\frac{\nu}{z} .
\end{gathered}
$$

Калибровочные преобразования безмассовых полей, относительно которых действие (3.6) инвариантно, определяются тем же соотношением, что и преобразование (2.14), где кет-вектор $|\Xi\rangle$ задает генерирующую форму параметров калибровочных преобразований. Для безмассовых полей в пространстве $\mathrm{AдC}_{d+1}$ этот кет-вектор зависит от грассмановой координаты $\theta$, осцилляторов $\alpha^{a}, \alpha^{z}, \eta, \rho$ и удовлетворяет тем же алгебраическим соотношениям, что и кет-вектор $|\Phi\rangle(3.3),(3.4)$ :

$$
|\Xi\rangle=\Xi\left(x, z, \theta, \alpha, \alpha^{z}, \eta, \rho\right)|0\rangle, \quad\left(N_{\alpha}+N_{z}+N_{\eta}+N_{\rho}-s\right)|\Xi\rangle=0, \quad \bar{\alpha}^{2}|\Xi\rangle=0 .
$$

Справедливы следующие замечания.

ЗАмечАниЕ 3.1. Как видно из формулы (3.11), оператор $M^{2}$ диагонален на пространстве кет-вектора $|\Phi\rangle$. Именно это обстоятельство является основной привлекательной чертой рассматриваемого в настоящей статье подхода, так как такое представление для $M^{2}$ позволяет легко решать уравнения движения, вычислять эффективное действие и тем самым исследовать АдС/КТП-соответствие. Например, применяя калибровку Зигеля $\left|\phi_{*}\right\rangle=0$ и интегрируя по грассмановой переменной $\theta$, мы приводим лагранжиан (3.6) к виду

$$
\mathcal{L}=\frac{1}{2}\left\langle\phi_{\mathrm{I}}\left|\left(\square-M^{2}\right)\right| \phi_{\mathrm{I}}\right\rangle-\frac{1}{2}\left\langle\phi_{\mathrm{II}}\left|\left(\square-M^{2}\right)\right| \phi_{\mathrm{II}}\right\rangle+\left\langle\bar{c}\left|\left(\square-M^{2}\right)\right| c\right\rangle .
$$


Лагранжиан (3.16) получен в работе [18] с помощью стандартной процедуры Фаддеева-Попова. Данный лагранжиан приводит к расщепляющимся уравнениям движения, что существенно упрощает исследование АдС/КТП-соответствия (см. работу [18]). Заметим, что при переходе от лагранжиана $\mathcal{L}(3.6)$ к лагранжиану $\mathcal{L}$ в выражении (3.16) мы применили замену $|\bar{c}\rangle \rightarrow-|\bar{c}\rangle$.

ЗАМЕЧАНИЕ 3.2. В работе [24] показано, что при использовании калибровки светового конуса безмассовое поле произвольного спина в пространстве $\mathrm{AдC}_{d+1}$ представимо в виде бесконечной прямой суммы массивных полей в пространстве $\mathbb{R}^{d-1,1}$. Применение построенной нами БРСТ-БВ-формулировки позволяет достаточно просто продемонстрировать справедливость этого факта для калибровочных безмассовых и массивных полей. С этой целью введем для кет-вектора $|\Phi\rangle(3.2)$ безмассового поля новое обозначение $\left|\Phi_{\mathrm{Ads}_{d+1}}(x, z)\right\rangle$, а кет-вектор $|\Phi\rangle(2.23)$ массивного поля массы $m$ после замены осциллятора $\zeta$ на $\alpha^{z}$ обозначим $\left|\Phi_{\mathbb{R}^{d-1,1}}(x, m)\right\rangle$. Теперь попытаемся связать кет-векторы $\left|\Phi_{\mathrm{Ads}_{d+1}}(x, z)\right\rangle$ и $\left|\Phi_{\mathbb{R}^{d-1,1}}(x, m)\right\rangle$, используя следующее преобразование Бесселя по радиальной переменной $z$ :

$$
\left|\Phi_{\mathrm{AdS}_{d+1}}(x, z)\right\rangle=\int_{0}^{\infty} d m Z_{\nu}(m z)(-1)^{N_{z}}\left|\Phi_{\mathbb{R}^{d-1,1}}(x, m)\right\rangle, \quad Z_{\nu}(z) \equiv \sqrt{z} J_{\nu}(z) .
$$

Докажем соотношение (3.17) следующим образом. Обозначим $G_{\mathrm{Ads}_{d+1}}$ операторы, приведенные в выражениях (3.8)-(3.11), а операторы, приведенные в выражениях (2.27)-(2.30), после замены осциллятора $\zeta$ на $\alpha^{z}$ обозначим $G_{\mathbb{R}^{d-1,1}}$. Для доказательства формулы (3.17) мы должны убедиться в справедливости следующих соотношений:

$$
G_{\mathrm{AdS}_{d+1}}\left|\Phi_{\mathrm{AdS}_{d+1}}(x, z)\right\rangle=\int_{0}^{\infty} d m Z_{\nu}(m z)(-1)^{N_{z}} G_{\mathbb{R}^{d-1,1}}\left|\Phi_{\mathbb{R}^{d-1,1}}(x, m)\right\rangle .
$$

Для операторов $M^{\eta a}, M^{\eta \eta}$, приведенных в формулах (2.27), (2.28) и (3.8), (3.9), соотношения (3.18) выполняюся очевидным образом. Для операторов $M^{\eta}, M^{2}$, приведенных в формулах $(2.29),(2.30)$ и $(3.10),(3.11)$, соотношения (3.18) можно легко доказать, если использовать следующие тождества для функции $Z_{\nu}(3.17)$ :

$\alpha^{z} \mathcal{T}_{\nu-1 / 2} Z_{\nu}=Z_{\nu} \alpha^{z}, \mathcal{T}_{-\nu+1 / 2} \bar{\alpha}^{z} Z_{\nu}=-Z_{\nu} \bar{\alpha}^{z}, \mathcal{T}_{\nu-1 / 2} Z_{\nu}=Z_{\nu-1}, \mathcal{T}_{-\nu-1 / 2} Z_{\nu}=-Z_{\nu+1}$,

где операторы $\nu, \mathcal{T}_{\nu}$ определены в выражениях (3.13), (3.15). Заметим, что оператор $M^{2}(3.11)$ допускает представление $M^{2}=-\mathcal{T}_{-\nu+1 / 2} \mathcal{T}_{\nu-1 / 2}$.

Реализация релятивистских симметрий. Релятивистские симметрии полей в пространстве Ад $_{d+1}$ описываются алгеброй $s o(d, 2)$. В рамках предлагаемого подхода только симметрии алгебры $s o(d-1,1)$ реализованы в явном виде, поэтому следует построить реализацию симметрий алгебры $s o(d, 2)$ на пространстве калибровочных полей $|\Phi\rangle(3.2)$, а также представить генераторы алгебры $s o(d, 2)$ в базисе алгебры $s o(d-1,1)$. В этом базисе алгебра $s o(d, 2)$ порождается суммой генераторов трансляций $P^{a}$, дилатации $D$, конформных бустов $K^{a}$ и генераторов алгебры $s o(d-1,1), J^{a b}$. Применим следующие коммутационные соотношения для данных генераторов:

$$
\begin{array}{cl}
{\left[D, P^{a}\right]=-P^{a},} & {\left[P^{a}, J^{b c}\right]=\eta^{a b} P^{c}-\eta^{a c} P^{b}, \quad\left[D, K^{a}\right]=K^{a},} \\
{\left[K^{a}, J^{b c}\right]=\eta^{a b} K^{c}-\eta^{a c} K^{b},} & {\left[P^{a}, K^{b}\right]=\eta^{a b} D-J^{a b}, \quad\left[J^{a b}, J^{c e}\right]=\eta^{b c} J^{a e}+3 \text { члена. }}
\end{array}
$$


Получим общую реализацию генераторов алгебры $s o(d, 2)$ на пространстве кет-векторов в рамках БРСТ-БВ-формализма:

$$
\begin{gathered}
P^{a}=\partial^{a}, \quad J^{a b}=x^{a} \partial^{b}-x^{b} \partial^{a}+M^{a b}, \quad D=x^{a} \partial^{a}+\Delta, \\
K^{a}=-\frac{1}{2} x^{2} \partial^{a}+x^{a} D+M^{a b} x^{b}+R^{a}, \\
\Delta \equiv z \partial_{z}+2 \theta \partial_{\theta}+M^{\eta \rho}+\frac{d-1}{2}, \\
R^{a}=-\frac{1}{2} z^{2} \partial^{a}-M^{z a} z+2 \theta M^{\rho a} .
\end{gathered}
$$

Операторы $M^{a b}, M^{z a}, M^{\eta \rho}, M^{\rho a}$ в формулах (3.19)-(3.21) не зависят от грассмановой переменной $\theta$, координат $x^{a}, z$ пространства АдС и производных $\partial_{\theta}, \partial^{a}, \partial_{z}$. Эти операторы зависят только от осцилляторов. Выпишем следующую реализацию операторов $M^{a b}, M^{z a}, M^{\eta \rho}, M^{\rho a}$ на пространстве кет-вектора (3.2):

$$
\begin{aligned}
& M^{a b}=\alpha^{a} \bar{\alpha}^{b}-\alpha^{b} \bar{\alpha}^{a}, \\
& M^{z a}=A^{z} \bar{\alpha}^{a}+A^{a} \bar{A}^{z}, \\
& M^{\eta \rho}=N_{\eta}-N_{\rho}, \\
& M^{\rho a}=\rho f_{\eta z} \bar{\alpha}^{a}+A^{a} \bar{f}_{\rho z} \bar{\rho},
\end{aligned}
$$

где оператор $A^{a}$ определен соотношением (3.12). В выражениях (3.23)-(3.25) использованы обозначения

$$
\begin{gathered}
A^{z} \equiv \alpha^{z} e_{\eta \rho z}+h_{z} \rho \eta \bar{\alpha}^{z}, \quad \bar{A}^{z} \equiv-e_{\eta \rho z} \bar{\alpha}^{z}-\alpha^{z} \bar{\eta} \bar{\rho} h_{z}, \\
f_{\rho z} \equiv\left[\frac{2 s+d-4-2 N_{z}-2 N_{\rho}}{2 s+d-4-2 N_{z}}\right]^{1 / 2}, \quad \bar{f}_{\eta z} \equiv\left[\frac{2 s+d-4-2 N_{z}-2 N_{\eta}}{2 s+d-4-2 N_{z}}\right]^{1 / 2}, \\
e_{\eta \rho z} \equiv e_{z}\left[\frac{\left(2 s+d-4-2 N_{z}\right)\left(2 s+d-4-2 N_{z}-2 N_{\eta}-2 N_{\rho}\right)}{\left(2 s+d-4-2 N_{z}-2 N_{\eta}\right)\left(2 s+d-4-2 N_{z}-2 N_{\rho}\right)}\right]^{1 / 2}, \\
h_{z} \equiv 2 e_{z}\left(\left(2 s+d-4-2 N_{z}\right)\left(2 s+d-6-2 N_{z}\right)\right)^{-1 / 2},
\end{gathered}
$$

где оператор $e_{z}$ определен формулой (3.14).

(. Симметрии алгебры $\$ \operatorname{osp}(\mathrm{d}-1,1 \mid 2) \$)$ Симметрии алгебры $\operatorname{ssp}(d-1,1 \mid 2)$ Из соотношений (3.10), (3.11) видно, что операторы $M^{2}, M^{\eta}$ зависят от радиальной координаты $z$ и от производной $\partial_{z}$, помимо осцилляторов. Поэтому, с одной стороны, рассмотрим спиновые операторы $M^{\eta a}, M^{\eta \eta}(3.8),(3.9)$, которые зависят только от осцилляторов. С другой стороны, мы видим, что в выражениях (3.19)-(3.21) для генераторов релятивистских симметрий появляются другие спиновые операторы $M^{\eta \rho}$, $M^{\rho a}$ и $M^{a b}$. Наше наблюдение состоит в том, что если к спиновым операторам $M^{\eta a}$, $M^{\eta \eta}$, которые появляются в выражении (3.7) для БРСТ-оператора, и спиновым операторам $M^{\eta \rho}, M^{\rho a}, M^{a b}$, которые появляются в выражениях (3.19)-(3.21), добавить спиновый оператор $M^{\rho \rho}$, определяемый соотношением $M^{\rho \rho}=\rho \bar{\rho}$, то полученное множество спиновых операторов образует супералгебру $\operatorname{sp}(d-1,1 \mid 2)$. Иначе говоря, мы имеем спиновые операторы, которые представимы следующим образом:

$$
\underbrace{M^{a b}}_{s o(d-1,1)}, \quad \underbrace{M^{\eta \rho}, M^{\eta \eta}, M^{\rho \rho}}_{s p(2)}, \quad \underbrace{M^{\eta a}, M^{\rho a}}_{\text {косет }}
$$


и удовлетворяют (анти)коммутационным соотношениям супералгебры $o s p(d-1,1 \mid 2)$ :

$$
\begin{aligned}
& {\left[M^{a b}, M^{c e}\right]=\eta^{b c} M^{a e}+3 \text { члена, } \quad\left[M^{\eta \rho}, M^{\eta \eta}\right]=2 M^{\eta \eta},} \\
& {\left[M^{\eta \rho}, M^{\rho \rho}\right]=-2 M^{\rho \rho}, \quad\left[M^{\eta \eta}, M^{\rho \rho}\right]=M^{\eta \rho}} \\
& {\left[M^{\eta \rho}, M^{\eta a}\right]=M^{\eta a}, \quad\left[M^{\eta \rho}, M^{\rho a}\right]=-M^{\rho a},} \\
& {\left[M^{\eta \eta}, M^{\rho a}\right]=M^{\eta a}, \quad\left[M^{\rho \rho}, M^{\eta a}\right]=M^{\rho a},} \\
& {\left[M^{\eta a}, M^{b c}\right]=\eta^{a b} M^{\eta c}-\eta^{a c} M^{\eta b}, \quad\left[M^{\rho a}, M^{b c}\right]=\eta^{a b} M^{\rho c}-\eta^{a c} M^{\rho b},} \\
& \left\{M^{\rho a}, M^{\eta b}\right\}=\eta^{a b} M^{\eta \rho}+M^{a b}, \quad\left\{M^{\eta a}, M^{\eta b}\right\}=-2 \eta^{a b} M^{\eta \eta}, \quad\left\{M^{\rho a}, M^{\rho b}\right\}=2 \eta^{a b} M^{\rho \rho} .
\end{aligned}
$$

Напомним, что при использовании (анти)коммутационных соотношений (3.26), (3.27) и понятия заряда $q$ для генератора $X$ супералгебры $\operatorname{sp} p(d-1,1 \mid 2)$, введенного соотношением $\left[M^{\eta \rho}, X\right]=q X$, супералгебра $\operatorname{ssp}(d-1,1 \mid 2)$ допускает разложение Кантора:

$$
\underbrace{M^{\rho \rho}}_{q=-2}, \quad \underbrace{M^{\rho a}}_{q=-1}, \quad \underbrace{M^{\eta \rho}, M^{a b}}_{q=0}, \quad \underbrace{M^{\eta a}}_{q=1}, \quad \underbrace{M^{\eta \eta}}_{q=2} .
$$

Поля, необходимые для построения БРСТ-БВ-формулировки, можно классифицировать по представлениям супералгебры $\operatorname{spp}(d-1,1 \mid 2)$. Этот факт хорошо известен для полей в пространстве $\mathbb{R}^{d-1,1}$, и он остается в силе для полей в пространстве $\mathrm{AдC}_{d+1}$. Также хорошо известно, что при построении БРСТ-БВ-формулировки полей в $\mathbb{R}^{d-1,1}$ используются спиновые операторы $M^{\eta a}, M^{\eta \eta}$, которые появляются в выражении (3.7) для БРСТ-оператора, и операторы $M^{a b}$, которые появляются в выражении (3.22) для генераторов алгебры Лоренца $s o(d-1,1)$. Операторы $M^{\eta a}$, $M^{\eta \eta}, M^{a b}$ образуют подалгебру супералгебры $o s p(d-1,1 \mid 2)$.

Как показывает наше исследование, ситуация в пространстве $\mathrm{AдC}_{d+1}$ выглядит более интересной. В этом случае при построении БРСТ-БВ-формулировки используются спиновые операторы $M^{\eta a}, M^{\eta \eta}$, которые появляются в выражении (3.12) для БРСТ-оператора, и операторы $M^{\eta \rho}, M^{\rho a}, M^{a b}$, которые появляются в выражениях (3.19)-(3.21) для генераторов алгебры релятивистских симметрий $s o(d, 2)$. Сами по себе спиновые операторы $M^{\eta a}, M^{\eta \eta}, M^{\eta \rho}, M^{\rho a}, M^{a b}$ не образуют супералгебру. Однако если к множеству этих спиновых операторов добавить оператор $M^{\rho \rho}=\rho \bar{\rho}$, который появляется в антикоммутаторе $M^{\rho a}(3.27)$, то мы получим супералгебру osp $(d-1,1 \mid 2)$. Заметим, что в выражении (3.21) для генераторов алгебры $s o(d, 2)$ оператор $M^{\rho a}$ появляется вместе с грассмановой переменной $\theta$. Поэтому последний антикоммутатор в (3.27) не дает вклада в коммутационные соотношения алгебры $s o(d, 2)$.

\section{4. ЗАКЛЮЧЕНИЕ}

В заключение отметим, что в настоящей статье мы построили БРСТ-БВ-формулировку свободных симметричных безмассовых полей в пространстве АдС. Предлагаемый подход может иметь интересные приложения при исследовании различных проблем теорий высших спинов в пространстве АдС. В частности, отметим проблему построения БРСТ-БВ-формулировки полей со смешанным типом симметрии [25]. 
Другая интересная проблема - построение БРСТ-БВ-формулировки теорий взаимодействующих полей высших спинов [26]-[28]. Применение БРСТ-БВ-подхода к конформным полям в пространстве АдС [29] также представляется интересной задачей.

\section{Список принятых обозначений}

\section{ПРИЛОЖЕНИЕ}

Мы считаем, что векторные индексы алгебры $s o(d-1,1)$ принимают следующие значения: $a, b, c, e=0,1, \ldots, d-1$. Чтобы упростить формулы, мы опускаем обозначение для плоской метрики $\eta^{a b}=(-,+, \ldots,+)$ в выражении для скалярного произведения: $X^{a} Y^{a} \equiv \eta_{a b} X^{a} Y^{b}$. Также мы используем грассманову переменную $\theta$, $\theta^{2}=0$. Ее левая производная и интеграл определяются как $\partial_{\theta}=\partial / \partial \theta, \int d \theta \theta=1$. Осцилляторами в настоящей работе названы операторы рождения $\alpha^{a}, \alpha^{z}, \zeta, \eta, \rho$ и соответствующие операторы уничтожения $\bar{\alpha}^{a}, \bar{\alpha}^{z}, \bar{\zeta}, \bar{\rho}, \bar{\eta}$.

(Анти)коммутационные соотношения, вакуум $|0\rangle$ и правила эрмитова сопряжения определяются соотношениями

$$
\begin{aligned}
& {\left[\bar{\alpha}^{a}, \alpha^{b}\right]=\eta^{a b}, \quad\left[\bar{\alpha}^{z}, \alpha^{z}\right]=1, \quad[\bar{\zeta}, \zeta]=1, \quad\{\bar{\rho}, \eta\}=1, \quad\{\bar{\eta}, \rho\}=1,} \\
& \bar{\alpha}^{a}|0\rangle=0, \quad \bar{\alpha}{ }^{z}|0\rangle=0, \quad \bar{\zeta}|0\rangle=0, \quad \bar{\eta}|0\rangle=0, \quad \bar{\rho}|0\rangle=0, \\
& \alpha^{a \dagger}=\bar{\alpha}^{a}, \quad \alpha^{z \dagger}=\bar{\alpha}^{z}, \quad \zeta^{\dagger}=\bar{\zeta}, \quad \eta^{\dagger}=\bar{\eta}, \quad \rho^{\dagger}=\bar{\rho} .
\end{aligned}
$$

Аналогичным образом осцилляторы $\alpha^{a}, \bar{\alpha}^{a}$ и $\alpha^{z}, \bar{\alpha}^{z}, \zeta, \bar{\zeta}, \eta, \bar{\rho}, \rho, \bar{\eta}$ преобразуются в векторных и скалярных представлениях алгебры $s o(d-1,1)$. Производные координат $x^{a}, z$ обозначаются как $\partial^{a} \equiv \eta^{a b} \partial / \partial x^{b}, \partial_{z} \equiv \partial / \partial z$ соответственно. Мы используем следующие сокращения для скалярных произведений осцилляторов и производных:

$$
\begin{aligned}
\square & \equiv \partial^{a} \partial^{a}, & \alpha^{2} \equiv \alpha^{a} \alpha^{a}, & \bar{\alpha}^{2} \equiv \bar{\alpha}^{a} \bar{\alpha}^{a}, \\
N_{\alpha} \equiv \alpha^{a} \bar{\alpha}^{a}, & N_{z} \equiv \alpha^{z} \bar{\alpha}^{z}, & N_{\zeta} \equiv \zeta \bar{\zeta}, & N_{\eta} \equiv \eta \bar{\rho}, \quad N_{\rho} \equiv \rho \bar{\eta} .
\end{aligned}
$$

Правила эрмитова сопряжения координат и производных определяются соотношениями $\left(x^{a}, z, \theta\right)^{\dagger}=\left(x^{a}, z, \theta\right),\left(\partial^{a}, \partial_{z}, \partial_{\theta}\right)^{\dagger}=\left(-\partial^{a},-\partial_{z}, \partial_{\theta}\right)$. Правило эрмитова сопряжения произведения двух операторов независимо от их грассмановой четности задается соотношением $(A B)^{\dagger}=B^{\dagger} A^{\dagger}$.

Духовые числа кет-векторов в формулах (2.5), (2.6) определяются как собственные значения внешнего оператора Фаддеева-Попова $\mathbf{N}_{\mathrm{FP}}^{\mathrm{ext}}$ на основе соотношения $\left(N_{\mathrm{FP}}^{\mathrm{int}}+\mathbf{N}_{\mathrm{FP}}^{\mathrm{ext}}\right)|\Phi\rangle=0$, где реализация внутреннего оператора Фаддеева-Попова $N_{\mathrm{FP}}^{\mathrm{int}}$ на кет-векторе $|\Phi\rangle(2.4)$ задается соотношением $N_{\mathrm{FP}}^{\text {int }}=\theta \partial_{\theta}+N_{\eta}-N_{\rho}$. Применяя его, получаем духовые числа кет-векторов в (2.5), (2.6):

$$
\begin{aligned}
& \operatorname{gh}\left(\left|\phi_{\mathrm{I}}\right\rangle\right)=0, \quad \operatorname{gh}(|c\rangle)=1, \quad \operatorname{gh}(|\bar{c}\rangle)=-1, \quad \operatorname{gh}\left(\left|\phi_{\text {II }}\right\rangle\right)=0, \\
& \operatorname{gh}\left(\left|\phi_{\mathrm{I} *}\right\rangle\right)=-1, \quad \operatorname{gh}\left(\left|c_{*}\right\rangle\right)=-2, \quad \operatorname{gh}\left(\left|\bar{c}_{*}\right\rangle\right)=0, \quad \operatorname{gh}\left(\left|\phi_{\mathrm{II} *}\right\rangle\right)=-1 .
\end{aligned}
$$

Так как духовые числа осцилляторов $\alpha^{a}, \alpha^{z}, \zeta$ и вакуума $|0\rangle$ считаются равными нулю, духовые числа кет-векторов (П.3) совпадают с духовыми числами тензорных полей, которые получаются при разложении кет-векторов (2.5), (2.6) в ряд по осцилляторам $\alpha^{a}, \alpha^{z}, \zeta$. Отметим, что духовые числа параметров калибровочных преобразований можно найти из соотношения $\left(N_{\mathrm{FP}}^{\mathrm{int}}+\mathbf{N}_{\mathrm{FP}}^{\mathrm{ext}}+1\right)|\Xi\rangle=0$. 
Бра-векторы $\langle\Phi|,\left\langle\phi_{\text {I,II }}\right|$ и кет-векторы $|\Phi\rangle,\left|\phi_{\text {I,II }}\right\rangle$ связаны операцией эрмитова сопряжения: $\langle\Phi|=(|\Phi\rangle)^{\dagger},\left\langle\phi_{\mathrm{I}, \mathrm{II}}\right|=\left(\left|\phi_{\mathrm{I}, \mathrm{II}}\right\rangle\right)^{\dagger}$. Бра-векторы и кет-векторы полей Фаддеева-Попова связаны соотношениями $\langle c|=| c\rangle^{\dagger},\langle\bar{c}|=-| \bar{c}\rangle^{\dagger}$, а для тензорных полей Фаддеева-Попова мы используем следующие правила эрмитова сопряжения: $c^{a_{1} \ldots a_{s^{\prime}} \dagger}=c^{a_{1} \ldots a_{s^{\prime}}}, \bar{c}^{a_{1} \ldots a_{s^{\prime}} \dagger}=-\bar{c}^{a_{1} \ldots a_{s^{\prime}}}$.

\section{Список литературы}

[1] C. Becchi, A. Rouet, R. Stora, Phys. Lett. B, 52 (1974), 344-346; И. В. Тютин, Калибровочная инвариантность в теории поля и статистической физике в операторной формулировке, Препринт ФИАН № 39, ФИАН, М., 1975, arXiv: 0812.0580.

[2] А. А. Славнов, ТMФ, 10:2 (1972), 153-161; J. C. Taylor, Nucl. Phys. B, 33:2 (1971), 436-444.

[3] W. Siegel, Phys. Lett. B, 149:1-3 (1984), 157-161; Phys. Lett. B, 151:5-6 (1985), 391-395; A. Neveu, P. C. West, Phys. Lett. B, 168:3 (1986), 192-200; H. Hata, K. Itoh, T. Kugo, H. Kunitomo, K. Ogawa, Phys. Rev. D, 34:8 (1986), 2360-2429.

[4] M. A. Vasiliev, Phys. Lett. B, 243:4 (1990), 378-382; M. A. Vasiliev, Phys. Lett. B, 567:1-2 (2003), 139-151, arXiv: hep-th/0304049.

[5] D. Z. Freedman, S. Mathur, A. Matusis, L. Rastelli, Nucl. Phys. B, 546:1-1 (1999), 96-118, arXiv: hep-th/9804058.

[6] H. Liu, A. A. Tseytlin, Nucl. Phys. B, 533:1-3 (1998), 88-108, arXiv: hep-th/9804083.

[7] R. R. Metsaev, Phys. Rev. D, 81:10 (2010), 106002, 29 pp., arXiv: 0907.4678.

[8] W. Mück, K.S. Viswanathan, Phys. Rev. D, 58:10 (1998), 106006, 7 pp., arXiv: hep-th/9805145.

[9] A. Polishchuk, JHEP, 07 (1999), 007, 12 pp., arXiv: hep-th/9905048.

[10] R. R. Metsaev, Phys. Rev. D, 85:12 (2012), 126011, 20 pp., arXiv: 1110.3749.

[11] R. R. Metsaev, Phys. Rev. D, 83:10 (2011), 106004, 28 pp., arXiv: 1011.4261.

[12] V.K. Dobrev, Nucl. Phys. B, 553:3 (1999), 559-582, arXiv: hep-th/9812194; N. Aizawa, V.K. Dobrev, Rep. Math. Phys., 75:2 (2015), 179-197, arXiv: 1406.2129.

[13] R. R. Metsaev, Phys. Rev. D, 78:10 (2008), 106010, 31 pp., arXiv: 0805.3472.

[14] R. R. Metsaev, JHEP, 10 (2015), 110, 52 pp., arXiv: 1507.06584.

[15] V.E. Didenko, E. D. Skvortsov, JHEP, 04 (2013), 158, 27 pp., arXiv: 1210.7963; O. A. Gelfond, M. A. Vasiliev, Nucl. Phys. B, 876:3 (2013), 871-917, arXiv: 1301.3123.

[16] I. Florakis, D. Sorokin, M. Tsulaia, Nucl. Phys. B, 890 (2014), 279-301, arXiv: 1408.6675; JHEP, 07 (2014), 105, 24 pp., arXiv: 1401.1645; X. Bekaert, J. Erdmenger, D. Ponomarev, C. Sleight, JHEP, 03 (2015), 170, 52 pp., arXiv: 1412.0016; JHEP, 11 (2015), 149, 57 pp., arXiv: 1508.04292; N. Boulanger, P. Kessel, E. D. Skvortsov, M. Taronna, Higher spin interactions in four dimensions: Vasiliev vs. Fronsdal, arXiv: 1508.04139.

[17] A.A. Tseytlin, Nucl. Phys. B, 877:2 (2013), 598-631, arXiv: 1309.0785; M. Beccaria, A. A. Tseytlin, J. Phys. A, 48:27 (2015), 275401, 33 pp., arXiv: 1503.08143; JHEP, 11 (2014), 114, 46 pp., arXiv: 1410.3273.

[18] Р. Р. Мецаев, ТМФ, 181:3 (2014), 495-514, arXiv: 1407.2601.

[19] R. R. Metsaev, Phys. Lett. B, 671:1 (2009), 128-134, arXiv: 0808.3945.

[20] R. R. Metsaev, Phys. Lett. B, 682:4-5 (2010), 455-461, arXiv: 0907.2207.

[21] A.K.H. Bengtsson, Class. Quantum Grav., 5:3 (1988), 437-452; Nucl. Phys. B, 333:2 (1990), 407-418.

[22] I. L. Buchbinder, A. Pashnev, M. Tsulaia, Phys. Lett. B, 523:3-4 (2001), 338-346, arXiv: hep-th/0109067; I. L. Buchbinder, V. A. Krykhtin, Nucl. Phys. B, 727:3 (2005), 537-563, arXiv: hep-th/0505092; I. L. Buchbinder, V.A. Krykhtin, P. M. Lavrov, Nucl. Phys. B, 762:3 (2007), 344-376, arXiv: hep-th/0608005. 
[23] A. Sagnotti, M. Tsulaia, Nucl. Phys. B, 682:1 (2004), 83-116, arXiv: hep-th/0311257; K. B. Alkalaev, M. Grigoriev, I. Tipunin, Nucl. Phys. B, 823:3 (2009), 509-545, arXiv: 0811.3999.

[24] R. R. Metsaev, Nucl. Phys. B, 563:1-2 (1999), 295-348, arXiv: hep-th/9906217.

[25] K. B. Alkalaev, M. Grigoriev, Nucl. Phys. B, 835:1 (2010), 197-220, arXiv: 0910.2690; Nucl. Phys. B, 853:3 (2011), 663-687, arXiv: 1105.6111; A. A. Reshetnyak, Phys. Part. Nucl., 41:6 (2010), 976-979, arXiv: 1002.0124; I. L. Buchbinder, A. Reshetnyak, Nucl. Phys. B, 862:1 (2012), 270-326, arXiv: 1110.5044; C. Burdik, A. Reshetnyak, J. Phys.: Conf. Ser., 343:1 (2012), 012102, 27 pp., arXiv: 1111.5516.

[26] N. Boulanger, S. Cnockaert, JHEP, 03 (2004), 031, 16 pp., arXiv: hep-th/0402180; X. Bekaert, N. Boulanger, S. Cnockaert, J. Math. Phys., 46:1 (2005), 012303, 33 pp., arXiv: hep-th/0407102.

[27] R. R. Metsaev, Phys. Lett. B, 720:1-3 (2013), 237-243, arXiv: 1205.3131.

[28] M. Henneaux, G. L. Gomez, R. Rahman, JHEP, 01 (2014), 087, 44 pp., arXiv: 1310.5152; JHEP, 08 (2012), 093, 43 pp., arXiv: 1206.1048; P. Dempster, M. Tsulaia, Nucl. Phys. B, 865:2 (2012), 353-375, arXiv: 1203.5597; M. Taronna, JHEP, 04 (2012), 029, 75 pp., arXiv: 1107.5843; I. Buchbinder, P. Dempster, M. Tsulaia, Nucl. Phys. B, 877:2 (2013), 260-289, arXiv: 1308.5539; I. L. Buchbinder, V. A. Krykhtin, Eur. Phys. J. C, 75 (2015), 454, 10 pp., arXiv: 1507.03723.

[29] R. R. Metsaev, Nucl. Phys. B, 885 (2014), 734-771, arXiv: 1404.3712.

Поступила в редакцию 24.08.2015 